\title{
Resection of a giant gastrointestinal stromal tumor after failed imatinib treatment during the COVID-19 pandemic: a case report
}

\author{
Katie Yusun Kwon ${ }^{1} \wedge$, Facundo Iriarte $^{2} \wedge$, Heather Ann Hartman ${ }^{1}$, Juhi Mittal ${ }^{3}$, Antonio Di Carlo ${ }^{1,4}$, \\ Abbas El-Sayed Abbas ${ }^{5}$ \\ ${ }^{1}$ Department of Surgery, Temple University Hospital, Philadelphia, PA, USA; ${ }^{2}$ Department of Thoracic Surgery, Fox Chase Cancer Center, \\ Philadelphia, PA, USA; ${ }^{3}$ Department of Medical Oncology, Fox Chase Cancer Center, Philadelphia, PA, USA; ${ }^{4}$ Department of Abdominal Organ \\ Transplant Surgery, Temple University Hospital, Philadelphia, PA, USA; ${ }^{5}$ Department of Thoracic Surgery, Lifespan Health System at Warren \\ Alpert Medical School, Brown University, Providence, RI, USA \\ Correspondence to: Katie Yusun Kwon. 9732 Natalies Way, Ellicott City, MD, USA. Email: kykwon13@gmail.com.
}

\begin{abstract}
Gastrointestinal stromal tumors (GISTs) can arise anywhere in the gastrointestinal tract, but predominately originate from the stomach. They are most commonly due to mutations in tyrosine kinase KIT or PDGFRA. While imatinib can be used as treatment for GISTs, the leading curative option is surgical resection, especially in the setting of primary resistance. In this case report, we describe an unusual history of a patient with a gastric GIST undergoing neoadjuvant imatinib therapy during 2020. Due to restrictions imposed by the pandemic, this patient had difficulty with in-person follow-up. After 2 months of imatinib therapy, the patient was contacted by voice-only telemedicine and reported massive abdominal distention and dyspnea. He was brought in urgently to the hospital. On exam and by computed tomography (CT) scan, the tumor appeared to have almost doubled in size, making it the largest GIST by volume reported in medical literature to date. The patient underwent an urgent laparotomy for complete resection of the tumor. Despite the exponential growth of the tumor, it remained attached only to the gastric body by a small stalk without invasion of neighboring structures. He was discharged without complications and has had no signs of recurrence at 6 months. This case reports highlights the importance of monitoring massive GIST tumors, despite imatinib therapy in the setting of the COVID-19 pandemic restrictions.
\end{abstract}

Keywords: Gastrointestinal stromal tumor (GIST); imatinib; telemedicine; COVID-19; case report

Received: 17 July 2021; Accepted: 11 November 2021; Published: 10 April 2022.

doi: 10.21037 /gist-21-12

View this article at: https://dx.doi.org/10.21037/gist-21-12

\section{Introduction}

Gastrointestinal stromal tumors (GISTs) are believed to arise from uncontrolled growth of interstitial cells of Cajal (1) and usually result from a gain-of-function mutation in receptor tyrosine kinase KIT (c-kit) or tyrosine kinase platelet-derived growth factor receptor $\alpha$ (PDGFRA) $(2,3)$. If the tumor is KIT negative, immunohistochemical staining for the discovered on GIST-1 (DOG1) antigen and CD34 is recommended and considered diagnostic (4).
The prognosis of GISTs is stratified using the consensus classification system from the National Institutes of Health (NIH) and is based on tumor size and mitotic count. High-risk GISTs are associated with poorer outcomes and tend to be larger in size with higher mitotic counts (5). Management of these tumors should depend on the size, location and presence of metastasis (6). Several studies report a benefit of neoadjuvant imatinib therapy for GISTs (7-9), but ultimately surgical resection is the main curative

^ ORCID: Katie Yusun Kwon, 0000-0002-2430-2455; Facundo Iriarte, 0000-0003-0027-5533; Heather Ann Hartman, 0000-0002-01644575 . 

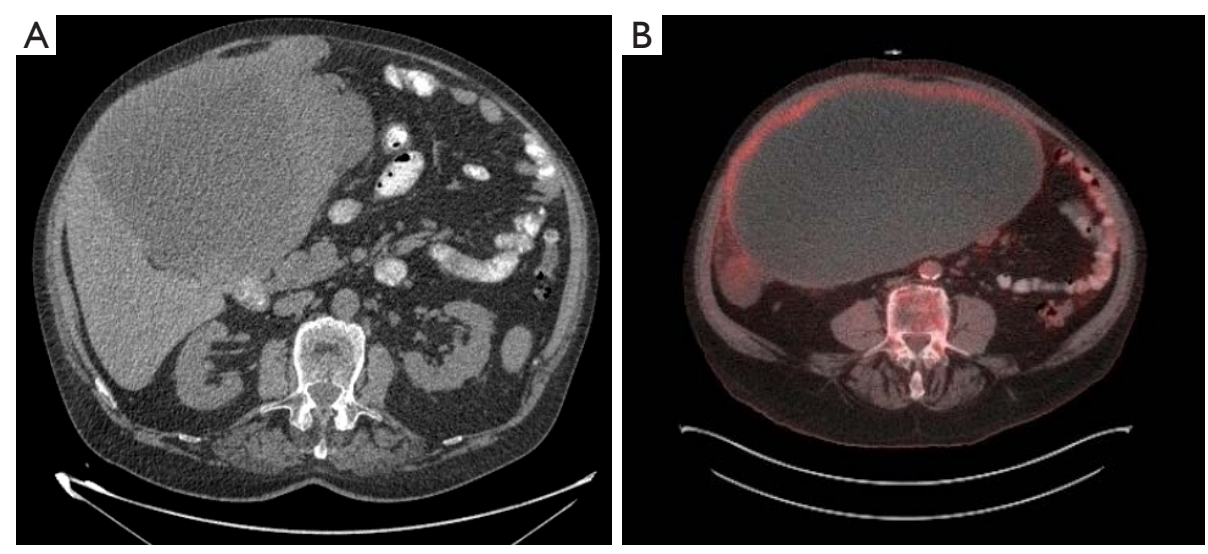

Figure 1 Initial CT of the GIST at the time of presentation, measuring $25.4 \mathrm{~cm} \times 17.3 \mathrm{~cm} \times 24.8 \mathrm{~cm}$ (A) and follow-up PET/CT imaging showing metabolic activity of the GIST, measuring $28.1 \mathrm{~cm} \times 16.9 \mathrm{~cm} \times 26 \mathrm{~cm}$, and right perihepatic lymph node (B). CT, computed tomography; GIST, gastrointestinal stromal tumor; PET, positron emission tomography.

option for large tumors (10). To date, the largest GIST reported in the medical literature was $42.0 \mathrm{~cm} \times 31.0 \mathrm{~cm}$ $\times 23.0 \mathrm{~cm}(11)$. Here we present an even larger GIST by volume measuring $23.9 \mathrm{~cm} \times 41.1 \mathrm{~cm} \times 37.9 \mathrm{~cm}$. Despite ongoing imatinib therapy, this tumor showed rapid and massive enlargement causing respiratory insufficiency requiring urgent surgical resection. Additionally, due to the restrictions from the COVID-19 pandemic, we were unable to monitor this dramatic growth as with traditional routine care. We present the following article in accordance with the CARE reporting checklist (available at https://gist. amegroups.com/article/view/10.21037/gist-21-12/rc).

\section{Case presentation}

A 70-year-old legally blind male with a medical history of oxygen-dependent chronic obstructive pulmonary disease and myocardial infarction underwent a lung cancer screening computed tomography (CT), which showed an incidental abdominal mass. His symptoms included abdominal discomfort and distension and on exam he showed mild abdominal swelling. A follow-up abdominal CT revealed a large heterogenous mass at the gastric antrum, measuring $25.4 \mathrm{~cm} \times 17.3 \mathrm{~cm} \times 24.8 \mathrm{~cm}$, with a centrally necrotic cystic component and appeared inseparable from the inferior hepatic margin (Figure 1A). Positron emission tomography showed the mass to be hypermetabolic with a standardized uptake value (SUV) of 5.5 and a right perihepatic lymph node with a SUV 4.5 of unclear significance (Figure 1B). Magnetic resonance imaging of the abdomen showed irregular arterial enhancement of the liver with delayed enhancement along the left hepatic lobe in segment II adjacent to the tumor with loss of tissue planes (Figure 2). The workup continued with an esophagogastroduodenoscopy and endoscopic ultrasound which showed no esophagogastric mucosal invasion. A fine needle aspiration biopsy demonstrated spindle cells with epithelioid morphology consistent with a GIST. Immunohistochemical stains were positive for DOG-1 and c-kit and negative for S100 and desmin.

After a multidisciplinary tumor board discussion, we elected to proceed with neo-adjuvant imatinib $400 \mathrm{mg}$ daily for 3 months, followed by restaging and reassessment for surgical resection due to the large size. In general, given the pandemic restrictions, there was a preference to telemedicine visits during this time with options for video and/or voice communication. The inability to arrange for in-person clinic visits lead to telehealth visits lacking a comprehensive physical assessment of our patient. About 8 months after the incidental finding, the patient began imatinib therapy and reported moderate improvement in some symptoms, but increased bloating, acid reflux, bilateral leg swelling and dyspnea on exertion with an increase in his home requirement of oxygen. Most of all, he noted significant increase in abdominal girth (Figure 3). As his symptoms continued to worsen, we promptly admitted him to the hospital about 2 months after initiation of imatinib therapy. Upon admission, physical exam showed tremendous enlargement of his abdomen causing shallow breathing. CT scan demonstrated massive interval growth of the tumor to $23.9 \mathrm{~cm} \times 41.1 \mathrm{~cm} \times 37.9 \mathrm{~cm}$ (Figure 4).

The patient underwent exploratory laparotomy with 

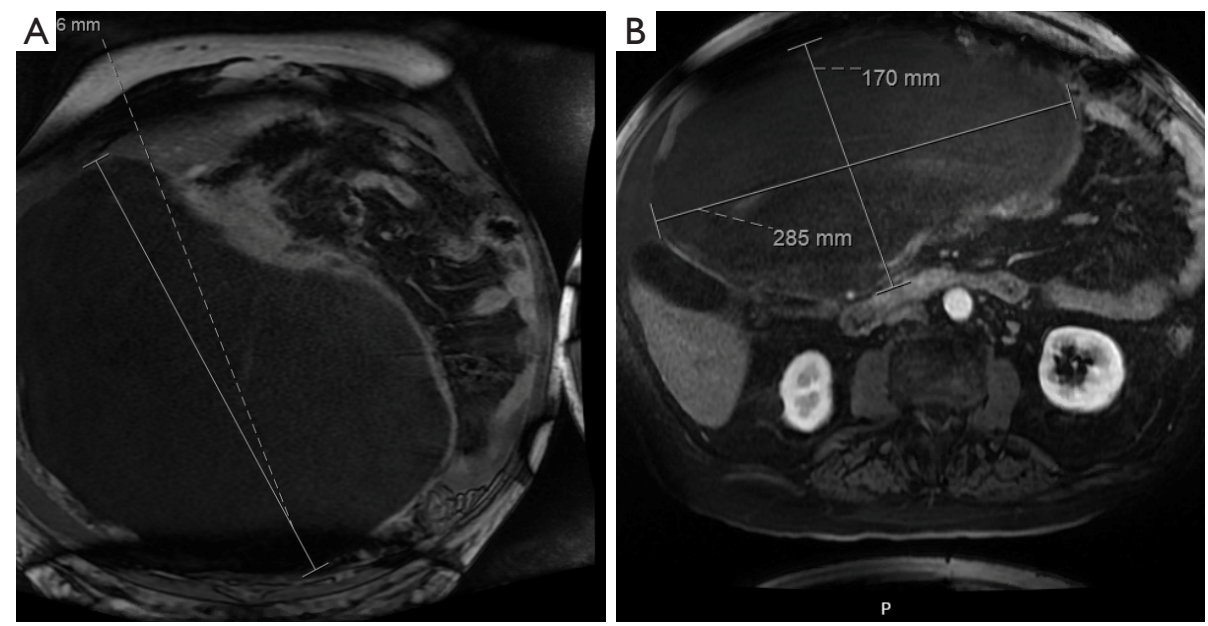

Figure 2 MRI reveals interval growth of the GIST in coronal view (A) and axial view (B). The GIST at this time measured $17 \mathrm{~cm} \times 28.5 \mathrm{~cm}$ $\times 28.8 \mathrm{~cm}$. MRI, magnetic resonance imaging; GIST, gastrointestinal stromal tumor.
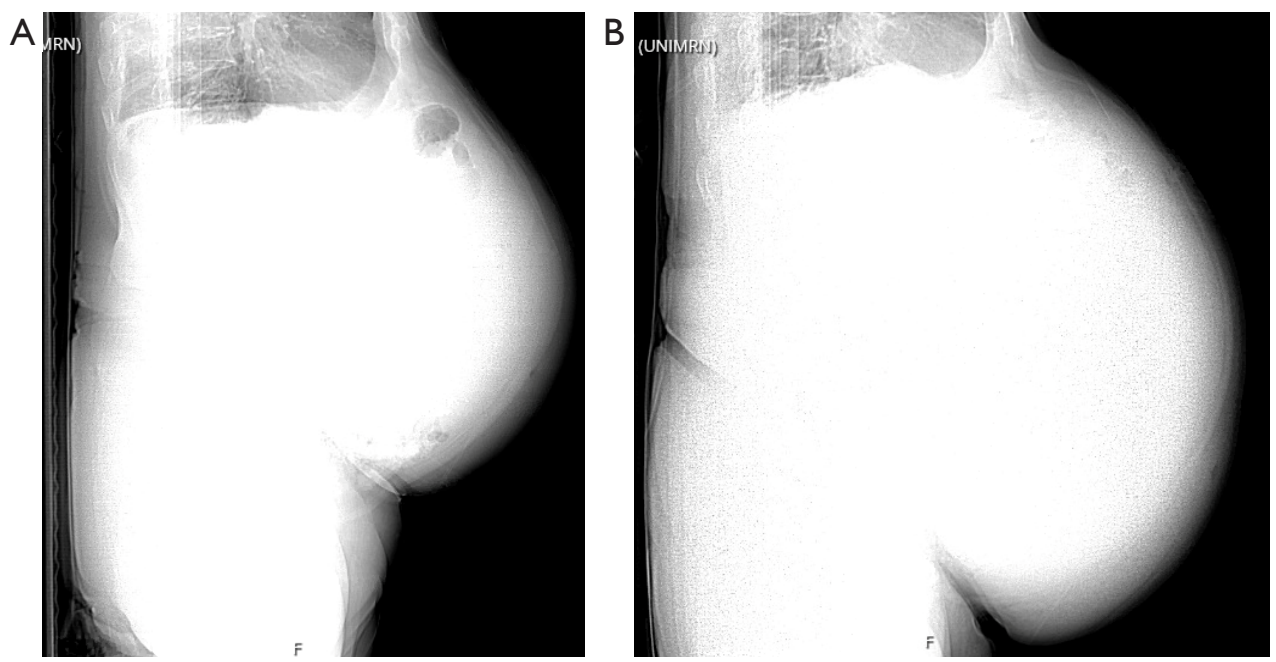

Figure 3 CT scan depicts the patient's silhouette at initial presentation (A) to just prior to surgical resection of the GIST (B). CT, computed tomography; GIST, gastrointestinal stromal tumor.

resection of the giant cystic mass, partial gastrectomy and omentectomy (Figure 5). Beginning with a careful midline laparotomy to avoid accidental rupture, we dissected laterally and drained the cystic portion about $40 \mathrm{~L}$ to facilitate the resection (Figure 6). Given the significantly large cystic volume of the mass, the lack of space in the lateral abdomen and the presumed heavy weight of the tumor, we believed controlled drainage was the safest approach. There was no evidence of hepatic or intestinal invasion and the mass was separated from the liver and bowel without difficulty. The mass appeared to arise from a small stalk attached to the antrum of the stomach, which necessitated a wedge partial gastrectomy (Figure 7). During the operation, the patient did exhibit mild hemodynamic instability requiring $700 \mathrm{~mL}$ of packed red blood cells, $2 \mathrm{~L}$ of lactated ringers, and $1 \mathrm{~L}$ of albumin $5 \%$.

Final pathology reported a high grade, epithelioid type GIST with variable mitoses (maximum $26 / 5 \mathrm{~mm}^{2}$ ) and necrosis (Figure 8). It was staged as pT4N0 with a negative gastric margin. Initially, the patient required blood and colloid transfusion for hemodynamic resuscitation postoperatively. However, the remainder of his post-operative 

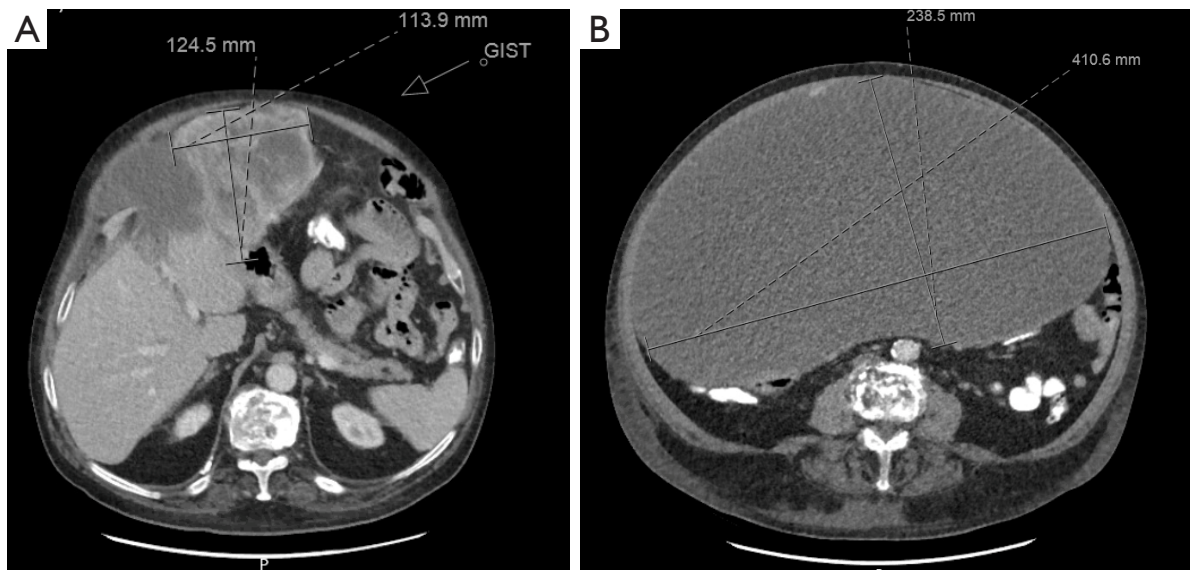

Figure 4 CT scan reveals enlargement of the tumor, measuring $23.9 \mathrm{~cm} \times 41.1 \mathrm{~cm} \times 37.9 \mathrm{~cm}$ (A) with a massive cystic component (B). CT, computed tomography.

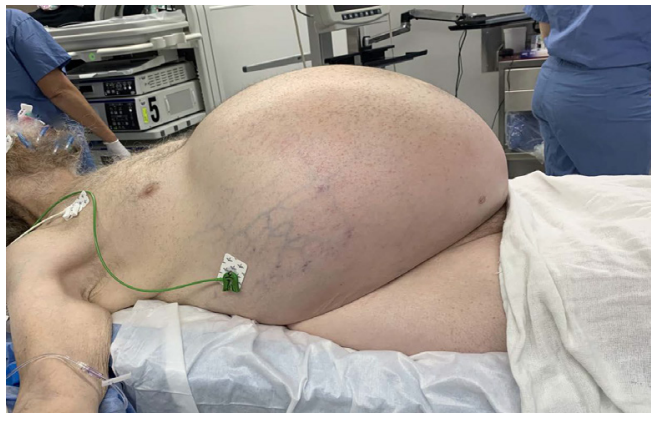

Figure 5 Patient is on the operating table, immediately prior to surgical resection. This image is published with the patient's consent.

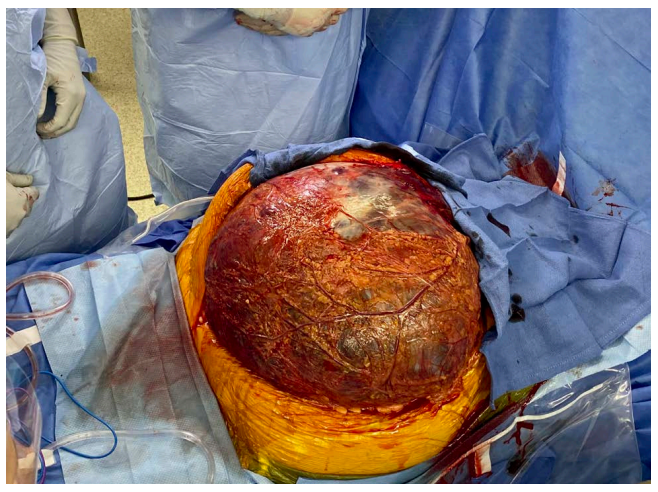

Figure 6 The initial incision, lateral dissection and exposure of the GIST. GIST, gastrointestinal stromal tumor. course was uncomplicated and he was discharged home on post-operative day 6 . Three weeks after the operation, he returned to clinic without issues. Imaging at 6 months showed no evidence of residual disease or metastasis. Next generation sequencing using the surgical specimen revealed an exon $18 \mathrm{D} 842 \mathrm{~V}$ activating mutation in the PDGFRA gene.

All procedures performed in this study were in accordance with the ethical standards of the institutional and/or national research committee(s) and with the Helsinki Declaration (as revised in 2013). Written informed consent was obtained from the patient for publication of this case report and accompanying images. A copy of the written consent is available for review by the editorial office of this journal.

\section{Discussion}

In 2002, the FDA approved the use of imatinib for treatment of GISTs due to structural similarities of kinase domains among KIT, PDGFRA and ABL (12). The efficacy rate of imatinib use with GISTs is around 53.8\%, including both complete and partial responses, while the disease-control rate, which adds stable disease, is $84 \%$ (13). However, $10-15 \%$ of GISTs are not responsive to imatinib (4). It is important to note that these response rates are calculated with relatively small GISTs. While some GISTs are found incidentally, symptomatic GISTs are a median size of $8.9 \mathrm{~cm}$, in a range of 1.0 to $35.0 \mathrm{~cm}$. Presenting symptoms can vary and are dependent on the location of the tumor. GISTs from the stomach can present 

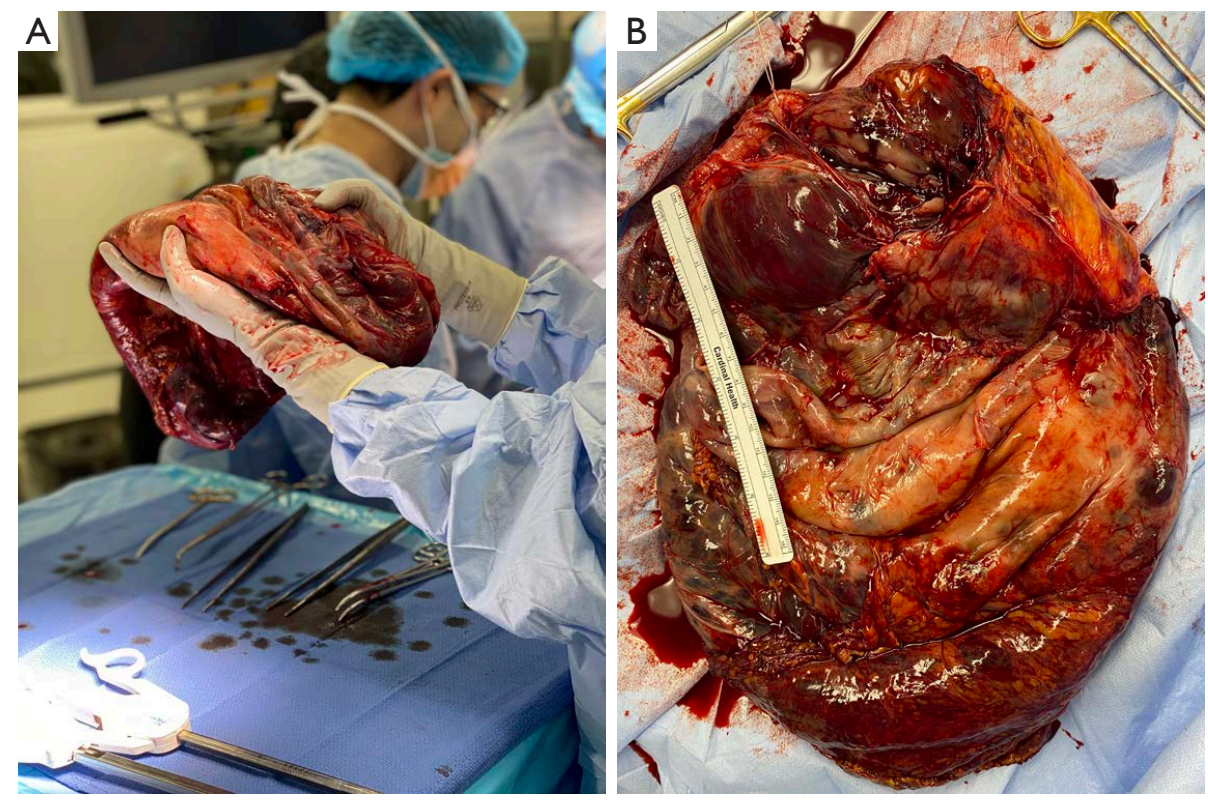

Figure 7 The gross specimen after the wedge partial gastrectomy and removal.
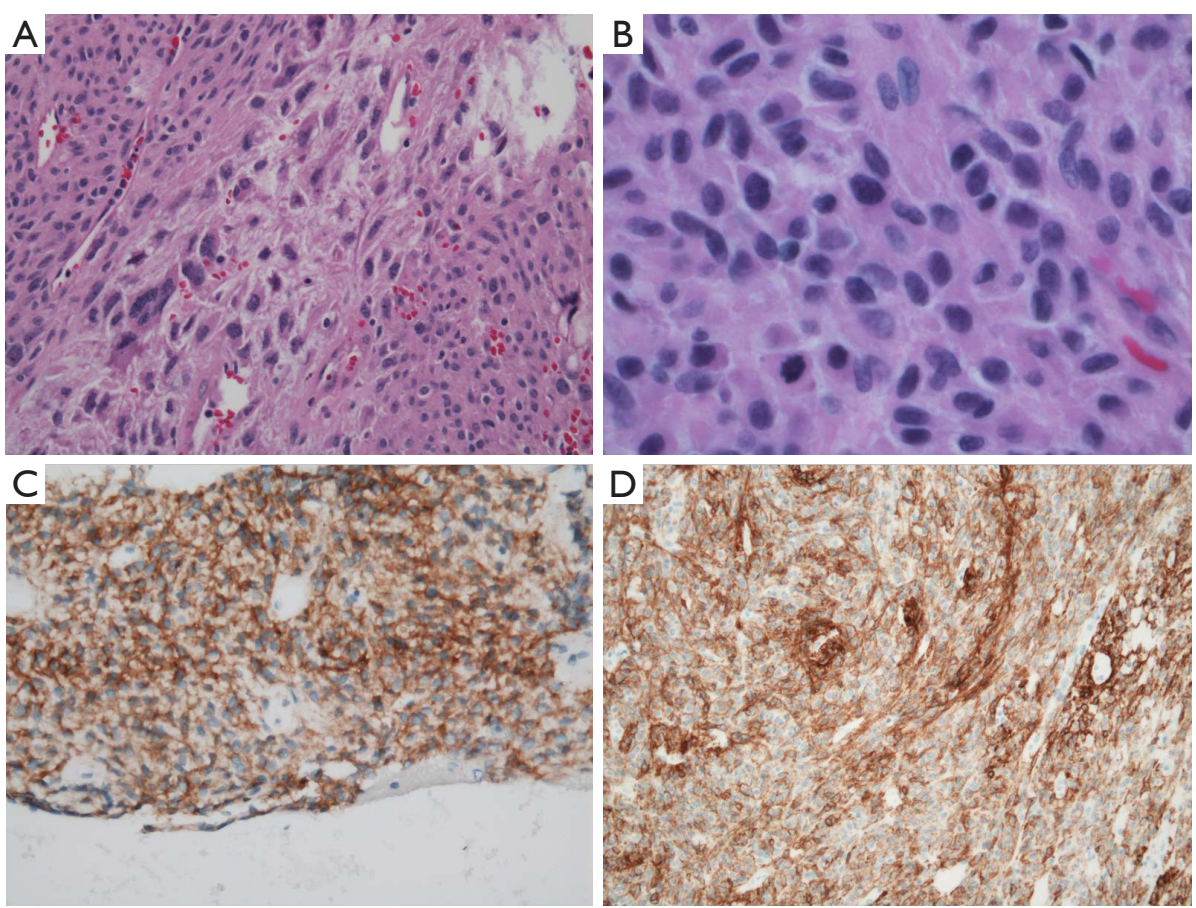

Figure 8 Histology of the resected surgical specimen showing high-grade epithelioid-type GIST at 20× magnitude (A, HE stain), 60× magnitude with mitosis (B, HE stain), DOG1 immunohistochemical staining at 20x magnitude (C) and CD117 immunohistochemical staining at 10× magnitude (D). GIST, gastrointestinal stromal tumor; DOG1, discovered on GIST-1. 
with early satiety, nausea, vomiting, dyspepsia, hematemesis and melena. Duodenal GISTs can present with ampullar obstruction and bleeding, while GISTs from the small bowel can present as bleeding, fatigue, abdominal pain and intestinal obstruction (14). For our patient, the vast enlargement of the GIST caused significant mass effect on both the abdominal and thoracic viscera, requiring urgent surgical resection.

Our case illustrates a rapidly evolving GIST despite imatinib therapy. Previous analyses reported imatinibassociated GIST shrinkage by 6-12 months (9). We plotted the course of this tumor according to the patient's imaging studies and fitted the data points with a second order polynomial curve $\left(R^{2}=0.9925\right)$, in order to illustrate the rapid, non-linear progression of the tumor (Figure S1). There was exponential growth coinciding with the initiation of imatinib therapy, indicating a resistance and an uncharacteristic period of growth. Our suspicions of primary resistance were confirmed by next generation sequencing of the surgical specimen, revealing a PDGFRA point mutation D $842 \mathrm{~V}$ in exon 18 . This mutation in addition to KIT exon 9-mutant tumors have been associated with primary imatinib resistance (15). While cystic changes may indicate disease response to imatinib through hemorrhage and necrosis of tissue $(16,17)$, to our knowledge, a concomitant increase in size has not been described. It is unclear why the size of this patient's GIST, which previously had been growing linearly, suddenly began expanding in a non-linear manner after the initiation of imatinib.

Using the NIH criteria, this GIST is stratified as highrisk. However, a case series of predominately cystic GISTs indicates that their behavior may be indolent with a lowrisk of malignancy and favorable prognosis (18). We were fortunate that the GIST was attached only to the gastric antrum by a small stalk and did not invade other organs. Despite the inability to obtain mutational analysis prior to the use of imatinib due to insufficient tissue quantity, the entire tumor was completely resected without residual disease. In addition, our patient's care was limited by the initial restrictions imposed by the COVID-19 pandemic, as his first scans were delayed due to the pause on all elective visits.

Our patient experienced multiple barriers to care due to the restrictions imposed by the COVID-19 pandemic. After the incidental finding of the GIST, his initial scan was delayed for about 4 months due to the cancellation of elective visits. Once he established care, the majority of this patient's visits were audio-only telehealth as the patient did not have access to a video conferencing device in addition to his visual disability. This led to the inability to monitor the unexpected rapid increase in the size of the mass. The pandemic led to a rapid transition to telemedicine, without ensuring equitable access for persons with disabilities, such as our patient. Vulnerable populations continue to experience challenges including access, transportation, and communication barriers $(19,20)$. Evaluating the impact of telemedicine on vulnerable populations is necessary to improve ongoing healthcare and to promote equity.

Furthermore, the enormous GIST negatively impacted our patient's quality of life. Prior to the operation, we spoke at length regarding his goals of treatment and the potential outcomes. He reported noticing significant growth of the mass coinciding with the start of imatinib therapy and therefore was eager to remove the tumor due to seemingly ineffective pharmacotherapy. He expressed sincere gratitude and relief after surgical resection of his giant abdominal mass.

This case report details the management of the largest GIST by volume reported in medical literature. Particularly with GISTs, it is important to be aware of the potential for primary resistance to imatinib, particularly when patients may not have access to in-person visits for monitoring of tumor growth. Herein we illustrated the complexity of management for a patient with visual impairment and a massive GIST that failed neoadjuvant imatinib therapy in the setting of the COVID-19 pandemic.

\section{Acknowledgments}

The authors thank Dr. Israh Akhtar for providing the histological findings of the resected specimens.

Funding: None.

\section{Footnote}

Reporting Checklist: The authors have completed the CARE reporting checklist. Available at https://gist.amegroups. com/article/view/10.21037/gist-21-12/rc

Conflicts of Interest: All authors have completed the ICMJE uniform disclosure form (available at https://gist.amegroups. com/article/view/10.21037/gist-21-12/coif). The authors have no conflicts of interest to declare.

Ethical Statement: The authors are accountable for all aspects of the work in ensuring that questions related to the accuracy or integrity of any part of the work are appropriately investigated and resolved. All procedures performed in 
this study were in accordance with the ethical standards of the institutional and/or national research committee(s) and with the Helsinki Declaration (as revised in 2013). Written informed consent was obtained from the patient for publication of this case report and accompanying images. A copy of the written consent is available for review by the editorial office of this journal.

Open Access Statement: This is an Open Access article distributed in accordance with the Creative Commons Attribution-NonCommercial-NoDerivs 4.0 International License (CC BY-NC-ND 4.0), which permits the noncommercial replication and distribution of the article with the strict proviso that no changes or edits are made and the original work is properly cited (including links to both the formal publication through the relevant DOI and the license). See: https://creativecommons.org/licenses/by-nc-nd/4.0/.

\section{References}

1. Kindblom LG, Remotti HE, Aldenborg F, et al. Gastrointestinal pacemaker cell tumor (GIPACT): gastrointestinal stromal tumors show phenotypic characteristics of the interstitial cells of Cajal. Am J Pathol 1998;152:1259-69.

2. Corless CL, Schroeder A, Griffith D, et al. PDGFRA mutations in gastrointestinal stromal tumors: frequency, spectrum and in vitro sensitivity to imatinib. J Clin Oncol 2005;23:5357-64.

3. Hirota S, Isozaki K, Nishida T, et al. Effects of loss-offunction and gain-of-function mutations of c-kit on the gastrointestinal tract. J Gastroenterol 2000;35 Suppl 12:75-9.

4. Nishida T, Blay JY, Hirota S, et al. The standard diagnosis, treatment, and follow-up of gastrointestinal stromal tumors based on guidelines. Gastric Cancer 2016;19:3-14.

5. Fletcher CD, Berman JJ, Corless C, et al. Diagnosis of gastrointestinal stromal tumors: a consensus approach. Int J Surg Pathol 2002;10:81-9.

6. Ahmed M. Recent advances in the management of gastrointestinal stromal tumor. World J Clin Cases 2020;8:3142-55.

7. Hiraki $Y$, Kato H, Shiraishi O, et al. Radical Resection of Huge Gastrointestinal Stromal Tumor of the Stomach Following Neoadjuvant Chemotherapy with lmatinib ACase Report. Gan To Kagaku Ryoho 2017;44:1723-5.

8. Hiramatsu Y, Konno H, Kamiya K, et al. A case of gastric GIST treated preoperatively by imatinib mesylate. Gan To Kagaku Ryoho 2004;31:1219-23.
9. Tang S, Yin Y, Shen C, et al. Preoperative imatinib mesylate (IM) for huge gastrointestinal stromal tumors (GIST). World J Surg Oncol 2017;15:79.

10. Wu PC, Langerman A, Ryan CW, et al. Surgical treatment of gastrointestinal stromal tumors in the imatinib (STI571) era. Surgery 2003;134:656-65; discussion 665-6.

11. Mohamed A, Botros Y, Hanna P, et al. Gigantic GIST: A Case of the Largest Gastrointestinal Stromal Tumor Found to Date. Case Rep Surg 2018;2018:6170861.

12. Mei L, Smith SC, Faber AC, et al. Gastrointestinal Stromal Tumors: The GIST of Precision Medicine. Trends Cancer 2018;4:74-91.

13. Kubota T. Gastrointestinal stromal tumor (GIST) and imatinib. Int J Clin Oncol 2006;11:184-9.

14. Nilsson B, Bümming P, Meis-Kindblom JM, et al. Gastrointestinal stromal tumors: the incidence, prevalence, clinical course, and prognostication in the preimatinib mesylate era--a population-based study in western Sweden. Cancer 2005;103:821-9.

15. Lee JH, Kim Y, Choi JW, et al. Correlation of imatinib resistance with the mutational status of KIT and PDGFRA genes in gastrointestinal stromal tumors: a meta-analysis. J Gastrointestin Liver Dis 2013;22:413-8.

16. Chourmouzi D, Sinakos E, Papalavrentios L, et al. Gastrointestinal stromal tumors: a pictorial review. J Gastrointestin Liver Dis 2009;18:379-83.

17. Levy AD, Remotti HE, Thompson WM, et al. Gastrointestinal stromal tumors: radiologic features with pathologic correlation. Radiographics 2003;23:283-304, 456; quiz 532.

18. Wang CZ, Hou YY, Shen KT, et al. Clinicopathological features and prognosis of cystic gastrointestinal stromal tumor. Zhonghua Wei Chang Wai Ke Za Zhi 2011;14:599-602.

19. Annaswamy TM, Verduzco-Gutierrez M, Frieden L. Telemedicine barriers and challenges for persons with disabilities: COVID-19 and beyond. Disabil Health J 2020;13:100973.

20. Calton B, Abedini N, Fratkin M. Telemedicine in the Time of Coronavirus. J Pain Symptom Manage 2020;60:e12-4.

doi: $10.21037 /$ gist-21-12

Cite this article as: Kwon KY, Iriarte F, Hartman HA, Mittal J, Di Carlo A, Abbas AE. Resection of a giant gastrointestinal stromal tumor after failed imatinib treatment during the COVID-19 pandemic: a case report. Gastrointest Stromal Tumor 2022;5:1. 


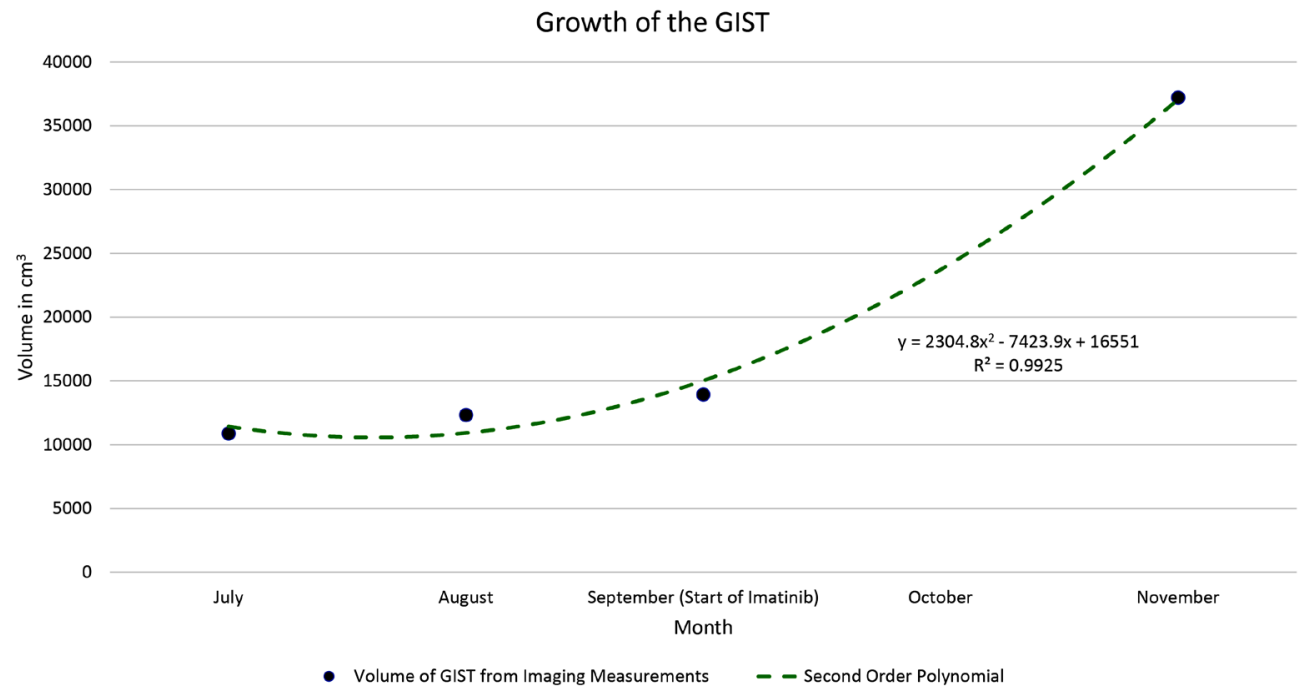

Figure S1 Illustrates the growth of the GIST from imaging measurements from the patient's initial presentation to immediately prior to surgical resection. Measurements were fitted with a second order polynomial $\left(\mathrm{R}^{2}=0.9925\right)$, demonstrating the non-linear growth of the GIST, particularly after the initiation of Imatinib in September. GIST, gastrointestinal stromal tumor. 